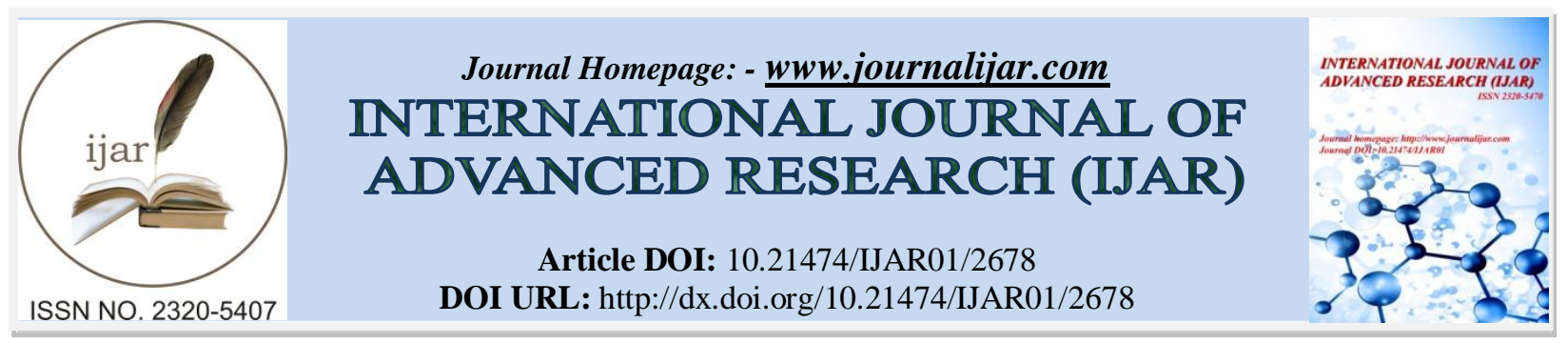

RESEARCH ARTICLE

\title{
RELATIONSHIP BETWEEN ADDICTION BEVERAGES THAT CONTAIN CAFFEINE AND ACADEMIC ACHIEVEMENT OF STUDENTS OF MEDICINE.
}

Bassem Dalil Falah Alosaimi, Yasir Saleh Abdulaziz Alsagaabi and khalid Yahya Namazi

\section{Manuscript Info}

Manuscript History

Received: 31 October 2016

Final Accepted: 01 December 2016

Published: December 2016

\section{Abstract}

This paper is about the full sized keyboard that is implemented using the image processing. In this type of keyboard the image of the keyboard is being projected on any plane surface and a pre calibrated camera tracks the movement of the fingers on the image and detects key being pressed. This keyboard uses embedded system and image processing to let the users work on any surface they want with a higher portability option. This technology has many application in various high tech and industrial sectors where the keyboards would be in sterile and low noise environments for example operation theatres.

Copy Right, IJAR, 2016,. All rights reserved.

\section{Introduction:-}

The purpose of this study is to explore the relationship between the caffeine consumption and the student GPA. Studying for an exam can be one of the most stressful events in a student's life. There are students who are always nervous and anxious and others find ways to deal with the burden. How do these students cope with stress? Some will exercise, and others procrastinate. When students find that stress and anxiety are overwhelming, they often seek alternate solutions. One of those solutions includes consuming a stimulant called caffeine. Caffeine relax the brain and they feel more attentive which allow them to study late night.

Caffeine consumption has become a huge controversy in the United States. There are differing opinions on caffeine, but most people tend to think that it affects a person negatively. Some people are beginning to label caffeine consumption as a social problem and some go as far as referring to the United States population as "caffeine addicts. Others are even pressing the regulation of the amount of caffeine in certain products (Troyer \& Markle, 1984).

Caffeine has been associated with occupational stress and anxiety about grade point average and is known to disrupt sleep quality and cause sleep disturbances (Gormley, 1996). It has also been associated with lower grades and depression in college students (Troyer \& Markle, 1984). However, with all of this negative attention, caffeine still seems to be the "drug" of choice. Four out of five Americans consume caffeine daily (Schardt \& Schmidt,1996). Many are consuming caffeine to have a heightened energy level or at least a sufficient one.

Caffeine is a stimulant and it works by interfering with the brain chemical adenosine. Adenosine usually slows down activity and has a calming effect. Higher consumption of caffeine results in lower levels of adenosine and this is why caffeine puts off sleep and raises concentration levels (Schardt \& Schmidt, 1996). College students have a great risk of becoming caffeine dependent because they can use these effects of caffeine to their advantage, especially when studying. Our strongest memories are of things that are emotionally provoking (Braun, 1996). That would mean that late night crammers armed with caffeine would tend to remember things more clearly because the caffeine is undoubtedly provoking their emotions. Studies have shown that caffeine improves the ability to do things that 
require "speed" but has little or no effect on things requiring "power" (Braun, 1996). When talking about things requiring "speed," it is meant that tasks such as simple arithmetic, visual-choice reaction time, and "data-driven" tasks are easier to do. Caffeine, then, would be ideal for a student preparing for a test that might require the use of these tasks. However, there is no solid evidence whether caffeine is or is not helpful in performing intellectual tasks. It has been shown that caffeine may worsen performance for more complicated tasks such as long word problems that are quite complex (Schardt \& Schmidt, 1996). This may be more along the lines of what is being studied by college students.

Studies conducted on this topic usually conclude that the effects of caffeine are dependent on the person. One study found signs that mental performance is affected by caffeine, and concluded that this affect was dependent on the impulsiveness of the user. Impulsive people were defined as those who were more concerned with speed than accuracy and who were more awake in the evening than in the morning. When impulsive people were given caffeine in the morning, when they were usually not fully aroused, such tasks as proofreading for grammatical and typographical errors improved. When caffeine was given to them in the evening, however, performance on tasks such as the above mentioned was worse than when they did not consume any caffeine at all. On the other hand, people who were not defined as impulsive reacted the exact opposite way, doing better in the evening and worse in the morning (Braun, 1996). This study basically contributed to the idea that the effects of caffeine are dependent on the person and also the task they are performing. Taking all of this into consideration, a general rule that could be formed seems to be caffeine is more helpful to people who tend to be not naturally aroused and for working on tasks that are straightforward (Braun, 1996). Therefore, caffeine would be an asset for a college student who is trying to put off sleep late at night to study for a test where memorization would be a pretty straightforward task. Studying this way, however, would be considered unhealthy according to a college survival tip list where studying during the day and avoiding food and drink containing caffeine right before or right after studying are listed (www.mtsu.edu/ studskl/10tips.html). This would be an unhealthy study habit because the best thing you could do before a test is sleep good the preceding night. Also, sleep deprivation in general is an unhealthy habit. This would probably cause a student to be more anxious about the test because they would be up all night worrying about it. This leads to the subject of anxiety level and caffeine consumption.

In another study, caffeine effects were measured in normal school-age children. Twenty-one participants were evaluated and measured on attention tests, short-term memory, processing speed, and anxiety levels and caffeine levels were determined by saliva samples. The study concluded that the children were reporting higher levels of anxiety after caffeine consumption but the caffeine enhanced their performance on a test of attention and motor tasks. Other data obtained from the same article also indicates that higher doses of caffeine produced a subjective feeling of anxiety in adults (Bernstein \& et al, 1994). Anxiety in a college student's life does not help them be as successful as they could be. According to Matiasen (1984), students with high levels of debilitating anxiety and lower levels of facilitating.

anxiety were linked to "academic frustration syndrome." This basically means that the high level of debilitating anxiety these students had was holding them back from their full potential and they wound up becoming frustrated with their academic work because they didn't have enough facilitating, or helpful anxiety to balance out. Caffeine dependent students may not even be aware that the caffeine they are consuming, for example to help them stay up late and study, may be having these negative effects on them.

Caffeine brings out mixed thoughts in people. Some think its helpful to them, others treat caffeine like it really is a "drug." Either way, it is known that caffeine has some sort of effect on a person. This study intended to see if there was any relationship between caffeine consumption and study habits. It was hypothesized that higher levels of caffeine consumption would be correlated with unhealthy study habits. It also tried to find out if college students are using caffeine to their advantage or disadvantage.

You may want to take a look at just how much caffeine you get in a typical day, especially if you're bothered by headaches, restlessness or anxiety. If your caffeine habit totals more than 500 milligrams $(\mathrm{mg})$ a day, you may want to consider cutting back.

With the growing popularity of energy drinks, many parents have become concerned about how much caffeine their kids are getting. The American Academy of Pediatrics recommends that adolescents get no more than $100 \mathrm{mg}$ of caffeine a day. Younger children shouldn't drink caffeinated beverages on a regular basis. 
The table below showing the caffeine content in popular drinks, sweets and medications.

\begin{tabular}{|c|c|c|c|c|c|c|c|c|c|}
\hline $\begin{array}{l}\text { Type of } \\
\text { coffee }\end{array}$ & Size* $^{*}$ & Caffeine $^{* *}$ & \multicolumn{2}{|l|}{ Type of tea } & Size* & Caffeine & $\begin{array}{l}\text { Soft } \\
\text { drink }\end{array}$ & Size* & Caffeine ${ }^{* *}$ \\
\hline $\begin{array}{l}\text { Espresso, } \\
\text { restaurant- } \\
\text { style }\end{array}$ & $\begin{array}{l}1 \quad \text { oz. } \\
(30 \\
m L)\end{array}$ & $40-75 \mathrm{mg}$ & \multicolumn{2}{|l|}{ Black tea } & $\begin{array}{l}8 \quad \text { oz. } \\
(240 \\
m L)\end{array}$ & $\begin{array}{l}\text { 14-61 } \\
\mathrm{mg}\end{array}$ & 7UP & $\begin{array}{l}12 \mathrm{oz} . \\
(355 \\
\mathrm{mL})\end{array}$ & $0 \mathrm{mg}$ \\
\hline $\begin{array}{l}\text { Espresso, } \\
\text { restaurant- } \\
\text { style, } \\
\text { decaffeinated }\end{array}$ & $\begin{array}{l}1 \quad \text { oz. } \\
(30 \\
m L)\end{array}$ & $0-15 \mathrm{mg}$ & \multicolumn{2}{|c|}{$\begin{array}{ll}\text { Black } & \text { tea, } \\
\text { decaffeinated } & \end{array}$} & $\begin{array}{l}8 \quad \text { oz. } \\
(240 \\
m L)\end{array}$ & $0-12 \mathrm{mg}$ & $\begin{array}{l}\text { A \& W } \\
\text { Root } \\
\text { Beer }\end{array}$ & $\begin{array}{l}12 \mathrm{oz} . \\
(355 \\
\mathrm{mL})\end{array}$ & $0 \mathrm{mg}$ \\
\hline $\begin{array}{l}\text { Generic } \\
\text { brewed }\end{array}$ & $\begin{array}{l}8 \quad \text { oz. } \\
(240 \\
m L)\end{array}$ & $95-200 \mathrm{mg}$ & \multicolumn{2}{|l|}{ Green tea } & $\begin{array}{l}8 \quad \text { oz. } \\
(240 \\
m L)\end{array}$ & $\begin{array}{l}24-40 \\
\mathrm{mg}\end{array}$ & $\begin{array}{l}\text { Barq's } \\
\text { Root } \\
\text { Beer }\end{array}$ & $\begin{array}{l}12 \mathrm{oz} . \\
(355 \\
\mathrm{mL})\end{array}$ & $18 \mathrm{mg}$ \\
\hline $\begin{array}{l}\text { Generic } \\
\text { brewed, } \\
\text { decaffeinated }\end{array}$ & $\begin{array}{l}8 \quad \text { oz. } \\
(240 \\
\mathrm{mL})\end{array}$ & $2-12 \mathrm{mg}$ & \multicolumn{2}{|c|}{$\begin{array}{l}\text { AriZona Iced Tea, } \\
\text { lemon-flavored }\end{array}$} & $\begin{array}{l}8 \quad \text { oz. } \\
(240 \\
m L)\end{array}$ & $11 \mathrm{mg}$ & $\begin{array}{l}\text { Coca- } \\
\text { Cola } \\
\text { Classic }\end{array}$ & $\begin{array}{l}12 \mathrm{oz} . \\
(355 \\
\mathrm{mL})\end{array}$ & $30-35 \mathrm{mg}$ \\
\hline $\begin{array}{l}\text { Generic } \\
\text { instant }\end{array}$ & $\begin{array}{l}8 \quad \text { oz. } \\
(240 \\
m L)\end{array}$ & $27-173 \mathrm{mg}$ & \multicolumn{2}{|c|}{$\begin{array}{l}\text { Generic instant, } \\
\text { unsweetened }\end{array}$} & $\begin{array}{l}8 \quad \text { oz. } \\
(240 \\
m L)\end{array}$ & $26 \mathrm{mg}$ & $\begin{array}{l}\text { Coca- } \\
\text { Cola } \\
\text { Zero }\end{array}$ & $\begin{array}{l}12 \mathrm{oz} . \\
(355 \\
\mathrm{mL})\end{array}$ & $35 \mathrm{mg}$ \\
\hline $\begin{array}{l}\text { Generic } \\
\text { instant, } \\
\text { decaffeinated }\end{array}$ & $\begin{array}{l}8 \quad \text { oz. } \\
(240 \\
m L)\end{array}$ & $2-12 \mathrm{mg}$ & \multicolumn{2}{|c|}{$\begin{array}{l}\text { Lipton Brisk } \\
\text { Lemon Iced Tea }\end{array}$} & $\begin{array}{l}8 \quad \text { oz. } \\
(240 \\
m L)\end{array}$ & $5-7 \mathrm{mg}$ & $\begin{array}{l}\text { Diet } \\
\text { Coke }\end{array}$ & $\begin{array}{l}12 \mathrm{oz} . \\
(355 \\
\mathrm{mL})\end{array}$ & $38-47 \mathrm{mg}$ \\
\hline $\begin{array}{l}\text { McDonald's } \\
\text { brewed }\end{array}$ & $\begin{array}{l}16 \mathrm{oz} . \\
(480 \\
\mathrm{mL})\end{array}$ & $100 \mathrm{mg}$ & & & & & $\begin{array}{l}\text { Diet } \\
\text { Pepsi }\end{array}$ & $\begin{array}{l}12 \mathrm{oz} . \\
(355 \\
\mathrm{mL})\end{array}$ & $27-37 \mathrm{mg}$ \\
\hline $\begin{array}{l}\text { McDonald's } \\
\text { Mocha } \\
\text { Frappe }\end{array}$ & $\begin{array}{l}16 \mathrm{oz} . \\
(480 \\
\mathrm{mL})\end{array}$ & $125 \mathrm{mg}$ & & & & & $\begin{array}{l}\text { Dr } \\
\text { Pepper }\end{array}$ & $\begin{array}{l}12 \mathrm{oz} . \\
(355 \\
\mathrm{mL})\end{array}$ & $36 \mathrm{mg}$ \\
\hline $\begin{array}{l}\text { Starbucks } \\
\text { Latte }\end{array}$ & $\begin{array}{l}16 \mathrm{oz} . \\
(480 \\
\mathrm{mL})\end{array}$ & $150 \mathrm{mg}$ & & & & & $\begin{array}{l}\text { Mountain } \\
\text { Dew }\end{array}$ & $\begin{array}{l}12 \mathrm{oz} . \\
(355 \\
\mathrm{mL})\end{array}$ & $46-55 \mathrm{mg}$ \\
\hline $\begin{array}{l}\text { Starbucks } \\
\text { Pike Place } \\
\text { brewed }\end{array}$ & $\begin{array}{l}16 \mathrm{oz} . \\
(480 \\
\mathrm{mL})\end{array}$ & $330 \mathrm{mg}$ & & & & & Pepsi & $\begin{array}{l}12 \mathrm{oz} . \\
(355 \\
\mathrm{mL})\end{array}$ & $32-39 \mathrm{mg}$ \\
\hline $\begin{array}{l}\text { Starbucks } \\
\text { Pike Place } \\
\text { brewed, } \\
\text { decaffeinated }\end{array}$ & $\begin{array}{l}16 \mathrm{oz} . \\
(480 \\
\mathrm{mL})\end{array}$ & $25 \mathrm{mg}$ & & & & & Sprite & $\begin{array}{l}12 \mathrm{oz} . \\
(355 \\
\mathrm{mL})\end{array}$ & $0 \mathrm{mg}$ \\
\hline Energy drink & \multicolumn{2}{|l|}{ Size* } & Caffeine** & \multicolumn{2}{|c|}{$\begin{array}{l}\text { Type of } \\
\text { product }\end{array}$} & Size & Caffeine* & & \\
\hline $\begin{array}{l}\text { 5-Hour } \\
\text { Energy }\end{array}$ & \multicolumn{2}{|c|}{2 oz. $(60 \mathrm{~mL})$} & $207 \mathrm{mg}$ & \multicolumn{2}{|c|}{$\begin{array}{l}\text { Chocolate } \\
\text { chips, } \\
\text { semisweet }\end{array}$} & 1 cup & $104 \mathrm{mg}$ & & \\
\hline $\begin{array}{l}\text { AMP, regular } \\
\text { or sugar-free }\end{array}$ & \multicolumn{2}{|c|}{$8 \mathrm{oz} .(240 \mathrm{~mL})$} & $72-74 \mathrm{mg}$ & \multicolumn{2}{|c|}{$\begin{array}{l}\text { Dark } \\
\text { chocolate- } \\
\text { coated coffee } \\
\text { beans }\end{array}$} & $\begin{array}{l}28 \\
\text { pieces }\end{array}$ & $336 \mathrm{mg}$ & & \\
\hline Cran-Energy & \multicolumn{2}{|c|}{$8 \mathrm{oz} .(240 \mathrm{~mL})$} & $70 \mathrm{mg}$ & $\begin{array}{l}\text { Foosh } \\
\text { Mints }\end{array}$ & Energy & $1 \mathrm{mint}$ & $100 \mathrm{mg}$ & & \\
\hline Full Throttle & \multicolumn{2}{|c|}{8 oz. $(240 \mathrm{~mL})$} & $70-72 \mathrm{mg}$ & \multicolumn{2}{|c|}{$\begin{array}{l}\text { Hershey's } \\
\text { Kisses }\end{array}$} & 9 pieces & $9 \mathrm{mg}$ & & \\
\hline Monster & \multicolumn{2}{|c|}{8 oz. $(240 \mathrm{~mL})$} & $80 \mathrm{mg}$ & \multicolumn{2}{|c|}{ Medications } & & & & \\
\hline Red Bull & \multicolumn{2}{|c|}{$8.4 \mathrm{oz}(250 \mathrm{~mL})$} & $76-80 \mathrm{mg}$ & \multicolumn{2}{|c|}{$\begin{array}{l}\text { Excedrin, } \\
\text { Extra Strength }\end{array}$} & 2 tablets & $130 \mathrm{mg}$ & & \\
\hline
\end{tabular}




\begin{tabular}{|l|l|l|l|l|l|l|}
\hline $\begin{array}{l}\text { Rockstar, } \\
\text { regular or } \\
\text { sugar-free }\end{array}$ & 8 oz. $(240 \mathrm{~mL})$ & $79-80 \mathrm{mg}$ & $\begin{array}{l}\text { NoDoz, } \\
\text { Maximum } \\
\text { Strength }\end{array}$ & 1 tablet & $200 \mathrm{mg}$ & \\
\hline $\begin{array}{l}\text { Vault, } \\
\text { regular or } \\
\text { sugar-free }\end{array}$ & 8 oz. $(240 \mathrm{~mL})$ & $47 \mathrm{mg}$ & & & \\
\hline
\end{tabular}

The goal form this study:-

The purposeofthisstudyis to look fora relationship betweenaddictionbeverages that containcaffeine andacademicachievementofstudentsofmedicineat the University ofImamMuhammadbinSaud Islamic Universityin Saudi Arabiain Riyadhanditisthis relationshiphasapositiveornegativeimpacton theachievementlevel of students.

\section{Material and methods:-}

This is correlation study to determine, describe and analyze relationships between caffeine consumption and the dependent variable which is the study grads or GPA. It tries to find out the relationship between the two variables. One variable is students caffeine intake and this was measured in cups or cans of caffeinated beverages students consume while study. Another variable is GPA which is know by the student or we get it from the academic office . To measure this this, a question is mentioned in questionnaire "when do you usually start preparing for the test?" and " how long do you study for exams per study session.

\section{Sample size:-}

The questionnaire will distributed among all male medical students in Al-Imam medical .

\section{Sample technique:-}

Convenient Sampling technique.

( Convenient sampling means to collect information from members of population who are conveniently available to provide it)

\section{Data coolection methods:-}

The questionnaire will be send through second year leader to all groups by emails.

Data management and analysis plane:-

Statistic software (SPSS) will be used to find out the result of this research.

\section{Ethical concentration:-}

Email already sent to the other but no replay yet

\section{Servey Questionnaire:-}

Purpose:-

To study the relationship between caffeine consumption and university students achievement grad . Direction: Please put check $(\sqrt{ })$ on the point that corresponds to what you are actually doing, thinking, and feeling regarding the statement. There are no right and wrong answers to this questionnaire. Rest assured that your answers will be treated in strictest and will be used only for this study.

\section{Gender:- \\ ○ M \\ $\circ \mathrm{F}$}

Age:-

○ $20-24$

- 25-30

- 30-35

- More than 35

\section{Marital Status:-}

- Single 
$\circ$ married

How would you describe your economic status?

○ Poor

- Below average

- Average

- Above average

o affluent

place of berth:-

○ Riyadh

○ Makkah

○ Medina

○ Qassim

- Eastern region

- Asir

○ Hail

- The northern border area

- Jezan

- Najran Other:

Approximate cumulative grade point average GPA: (choose one):-

○ $4-5$

○ $3.99-3.00$

○ $2.99-2.00$

○ $1.99-1$

- Below 1

Size of your household, i.e. the number of people, including yourself, who live in your house/dwelling for at least three months of the year
$\circ$ alone
○ $2-3$
○ $4-5$
○ More than 5

Drink caffeine as on the following contain:-

\begin{tabular}{|l|l|l|l|l|}
\hline Drinks & always & often & seldom & never \\
\hline $\begin{array}{l}\text { caffeinatedcoffee: Turkish coffee, espressoArabic coffee, } \\
\text { Americana, French coffee, hot chocolate, mocha, latte, } \\
\text { cappuccino, conpana, machiata, Nescafe. }\end{array}$ & & & & \\
\hline Tea drinks: Red tea, English tea, block tea, tea with milk. & & & & \\
\hline soft drinks: Pepsi, coca cola, B cola & & & & \\
\hline $\begin{array}{l}\text { energydrinks: Red boll, Bison, Power horse, Bugzy, Red } \\
\text { Rockek. }\end{array}$ & & & & \\
\hline
\end{tabular}

How much do you drinks per day from this caffeinated drink:-

○ $<200 \mathrm{ml}$

○ $200-400 \mathrm{ml}$

○ $>400 \mathrm{ml}$

why you drinks this caffeinated drinks:-

- Due to Social life

- Due to placement

- Due to studying concentration 
- Due to physical headache

- Due to stressful situation

- Due to eat meal

Other:.

\section{Do you feel any of this symptoms when stop this Caffeinated Drinks:-}

○ Insomnia

- Nervousness

- Restlessness

- Irritability

- Stomach upset

- Fast heartbeat

\section{Muscle tremors Other:-}

\begin{tabular}{|c|c|c|}
\hline \multicolumn{3}{|l|}{ Notes } \\
\hline \multicolumn{2}{|l|}{ Output Created } & 15-JAN-2014 13:33:25 \\
\hline \multicolumn{2}{|l|}{ Comments } & \\
\hline \multirow[t]{5}{*}{ Input } & $\begin{array}{l}\text { Active } \\
\text { Dataset }\end{array}$ & DataSet1 \\
\hline & Filter & <none $>$ \\
\hline & Weight & $<$ none $>$ \\
\hline & Split File & <none $>$ \\
\hline & $\begin{array}{l}\mathrm{N} \text { of } \\
\text { Rows in } \\
\text { Working } \\
\text { Data File }\end{array}$ & 91 \\
\hline \multirow[t]{2}{*}{ Missing Value Handling } & $\begin{array}{l}\text { Definition } \\
\text { of } \\
\text { Missing }\end{array}$ & User-defined missing values are treated as missing. \\
\hline & $\begin{array}{l}\text { Cases } \\
\text { Used }\end{array}$ & Statistics are based on all cases with valid data. \\
\hline \multicolumn{2}{|l|}{ Syntax } & $\begin{array}{l}\text { FREQUENCIES VARIABLES=Age GENDER } \\
\text { MaritalStatus } \\
\text { Howwouldyoudescribeyoureconomicstatus } \\
\text { ApproximatecumulativegradepointaverageGPAchooseone } \\
\text { Sizeofyourhouseholdi.e.thenumberofpeopleincludingyourselfwholive } \\
\text { Idrinkcaffeineasonthefollowingcontain } \\
\text { Idrinkcaffeineasonthefollowingcontain_A } \\
\text { Idrinkcaffeineasonthefollowingcontain_B } \\
\text { Idrinkcaffeineasonthefollowingcontain_C } \\
\text { howmuchdoyoudrinksperdayfromthiscaffeinateddrink } \\
\text { whyyoudrinksthiscaffeinateddrinks } \\
\text { doyoufeelanyofthissymptomswhenstopthiscaffeinateddrinks } \\
\text { /NTILES=4 } \\
\text { /STATISTICS=STDDEV VARIANCE RANGE MINIMUM } \\
\text { MAXIMUM MEAN MEDIAN MODE } \\
\text { /BARCHART FREQ } \\
\text { /ORDER=ANALYSIS. }\end{array}$ \\
\hline \multirow[t]{2}{*}{ Resources } & $\begin{array}{l}\text { Processor } \\
\text { Time }\end{array}$ & 00:00:02.10 \\
\hline & $\begin{array}{l}\text { Elapsed } \\
\text { Time }\end{array}$ & 00:00:03.00 \\
\hline
\end{tabular}


[DataSet1]

\begin{tabular}{|c|c|c|c|c|c|c|c|c|}
\hline \multicolumn{9}{|c|}{ Statistics } \\
\hline & & Age & GENDER & $\begin{array}{l}\text { Marital } \\
\text { Status }\end{array}$ & $\begin{array}{l}\text { How } \\
\text { would } \\
\text { you } \\
\text { describe } \\
\text { your } \\
\text { economic } \\
\text { status }\end{array}$ & $\begin{array}{l}\text { place } \\
\text { of } \\
\text { berth }\end{array}$ & $\begin{array}{l}\text { Approximate } \\
\text { cumulative } \\
\text { grade point } \\
\text { average } \\
\text { GPA: } \\
\text { (choose one) }\end{array}$ & $\begin{array}{l}\text { Size of your } \\
\text { household, i.e. } \\
\text { the number of } \\
\text { people, } \\
\text { including } \\
\text { yourself, who } \\
\text { live in your } \\
\text { house/dwelling } \\
\text { for at least } \\
\text { three months } \\
\text { of the year }\end{array}$ \\
\hline \multirow[t]{2}{*}{$\mathrm{N}$} & Valid & 91 & 91 & 91 & 91 & 91 & 91 & 91 \\
\hline & Missing & 0 & 0 & 0 & 0 & 0 & 0 & 0 \\
\hline
\end{tabular}

\begin{tabular}{|c|c|c|c|c|c|c|c|}
\hline \multicolumn{8}{|c|}{ Statistics } \\
\hline & & $\begin{array}{l}\text { I drink } \\
\text { caffeine as } \\
\text { on the } \\
\text { following } \\
\text { contain: }\end{array}$ & $\begin{array}{l}\text { I drink } \\
\text { caffeine as } \\
\text { on the } \\
\text { following } \\
\text { contain: }\end{array}$ & $\begin{array}{l}\text { I drink } \\
\text { caffeine as } \\
\text { on the } \\
\text { following } \\
\text { contain: }\end{array}$ & $\begin{array}{l}\text { I drink } \\
\text { caffeine as } \\
\text { on the } \\
\text { following } \\
\text { contain: }\end{array}$ & $\begin{array}{l}\text { how much } \\
\text { do you } \\
\text { drinks per } \\
\text { day from } \\
\text { this } \\
\text { caffeinated } \\
\text { drink: }\end{array}$ & $\begin{array}{l}\text { why you } \\
\text { drinks this } \\
\text { caffeinated } \\
\text { drinks }\end{array}$ \\
\hline \multirow[t]{2}{*}{$\mathrm{N}$} & Valid & 91 & 91 & 91 & 91 & 91 & 91 \\
\hline & Missing & 0 & 0 & 0 & 0 & 0 & 0 \\
\hline
\end{tabular}

\begin{tabular}{|l|l|l|}
\hline \multicolumn{2}{|l|}{ Statistics } & $\begin{array}{l}\text { do you feel any of this symptoms when stop this } \\
\text { caffeinated drinks }\end{array}$ \\
\hline \multirow{2}{*}{$\mathrm{N}$} & Valid & 91 \\
\cline { 2 - 3 } & Missing & 0 \\
\hline
\end{tabular}

\section{Frequency Table}

\begin{tabular}{|l|l|l|l|l|l|}
\hline \multicolumn{2}{|l|}{ Age } & Frequency & Percent & Valid Percent & $\begin{array}{l}\text { Cumulative } \\
\text { Percent }\end{array}$ \\
\hline \multirow{3}{*}{ Valid } & & 1 & & & 1.1 \\
\cline { 2 - 6 } & $20-25$ & 70 & 1.1 & 1.1 & 78.0 \\
\cline { 2 - 6 } & $25-30$ & 10 & 76.9 & 76.9 & 89.0 \\
\cline { 2 - 6 } & $30-35$ & 8 & 11.0 & 11.0 & 97.8 \\
\cline { 2 - 6 } & More than 35 & 2 & 2.2 & 8.8 & 100.0 \\
\cline { 2 - 6 } & Total & 91 & 100.0 & 100.0 & \\
\hline
\end{tabular}

\begin{tabular}{|l|l|l|l|l|l|}
\hline \multicolumn{2}{|l|}{ GENDER } & Frequency & Percent & Valid Percent & $\begin{array}{l}\text { Cumulative } \\
\text { Percent }\end{array}$ \\
\hline \multirow{2}{*}{ Valid } & & 3 & 3.3 & 3.3 & 3.3 \\
\cline { 2 - 6 } & male & 88 & 96.7 & 96.7 & 100.0 \\
\cline { 2 - 6 } & Total & 91 & 100.0 & 100.0 & \\
\hline
\end{tabular}




\begin{tabular}{|c|c|c|c|c|c|}
\hline \multicolumn{6}{|c|}{ Marital Status } \\
\hline & & Frequency & Percent & Valid Percent & Cumulative Percent \\
\hline \multirow[t]{4}{*}{ Valid } & & 4 & 4.4 & 4.4 & 4.4 \\
\hline & Married & 17 & 18.7 & 18.7 & 23.1 \\
\hline & Single & 70 & 76.9 & 76.9 & 100.0 \\
\hline & Total & 91 & 100.0 & 100.0 & \\
\hline
\end{tabular}

\begin{tabular}{|l|l|l|l|l|l|}
\hline \multicolumn{2}{|l|}{ How would you describe your economic status } \\
\hline \multicolumn{1}{|c|}{} & Frequency & Percent & Valid Percent & $\begin{array}{l}\text { Cumulative } \\
\text { Percent }\end{array}$ \\
\hline \multirow{3}{*}{ Valid } & & 2 & 2.2 & 2.2 & 2.2 \\
\cline { 2 - 6 } & Above average & 15 & 16.5 & 16.5 & 18.7 \\
\cline { 2 - 6 } & affluent & 5 & 5.5 & 5.5 & 24.2 \\
\cline { 2 - 6 } & Average & 68 & 74.7 & 74.7 & 98.9 \\
\cline { 2 - 6 } & Poor & 1 & 1.1 & 1.1 & 100.0 \\
\cline { 2 - 6 } & Total & 91 & 100.0 & 100.0 & \\
\hline
\end{tabular}

\begin{tabular}{|c|c|c|c|c|c|}
\hline \multicolumn{6}{|c|}{ place of berth } \\
\hline & & Frequency & Percent & Valid Percent & $\begin{array}{l}\text { Cumulative } \\
\text { Percent }\end{array}$ \\
\hline \multirow[t]{13}{*}{ Valid } & & 4 & 4.4 & 4.4 & 4.4 \\
\hline & Al baha & 1 & 1.1 & 1.1 & 5.5 \\
\hline & Eastern region & 7 & 7.7 & 7.7 & 13.2 \\
\hline & gizan & 1 & 1.1 & 1.1 & 14.3 \\
\hline & Jeddah & 1 & 1.1 & 1.1 & 15.4 \\
\hline & Makkah & 3 & 3.3 & 3.3 & 18.7 \\
\hline & Medina & 4 & 4.4 & 4.4 & 23.1 \\
\hline & Qassim & 13 & 14.3 & 14.3 & 37.4 \\
\hline & Riyadh & 54 & 59.3 & 59.3 & 96.7 \\
\hline & tabuk & 1 & 1.1 & 1.1 & 97.8 \\
\hline & جيزان & 1 & 1.1 & 1.1 & 98.9 \\
\hline & عسبر & 1 & 1.1 & 1.1 & 100.0 \\
\hline & Total & 91 & 100.0 & 100.0 & \\
\hline
\end{tabular}

\begin{tabular}{|l|l|l|l|l|l|}
\hline \multicolumn{2}{|c|}{ Approximate cumulative grade point average GPA: (choose one) } \\
\hline \multirow{4}{*}{ Valid } & Frequency & Percent & Valid Percent & Cumulative Percent \\
\cline { 2 - 6 } & $4-5$ & 3 & 3.3 & 3.3 & 3.3 \\
\cline { 2 - 6 } & $1.99-1$ & 18 & 19.8 & 19.8 & 23.1 \\
\cline { 2 - 6 } & $2.99-2$ & 22 & 1.1 & 1.1 & 24.2 \\
\cline { 2 - 6 } & $3.99-3$ & 47 & 24.2 & 24.2 & 48.4 \\
\cline { 2 - 6 } & Total & 91 & 51.6 & 51.6 & \\
\hline
\end{tabular}

Size of your household, i.e. the number of people, including yourself, who live in your house/dwelling for at least three months of the year

\begin{tabular}{|l|l|l|l|l|l|}
\hline \multicolumn{2}{|c|}{} & Frequency & Percent & Valid Percent & Cumulative Percent \\
\hline Valid & 3 & 3.3 & 3.3 & 3.3 \\
\cline { 2 - 6 } & $2-3$ & 24 & 26.4 & 26.4 & 29.7 \\
\cline { 2 - 6 } & $4-5$ & 30 & 33.0 & 33.0 & 62.6 \\
\cline { 2 - 6 } & alone & 3 & 3.3 & 3.3 & 65.9 \\
\cline { 2 - 6 } & More than 5 & 31 & 34.1 & 34.1 & 100.0 \\
\cline { 2 - 6 } & Total & 91 & 100.0 & 100.0 & \\
\hline
\end{tabular}




\begin{tabular}{|c|c|c|c|c|c|}
\hline \multicolumn{6}{|c|}{ how much do you drinks per day from this caffeinated drink : } \\
\hline & & Frequency & Percent & Valid Percent & $\begin{array}{l}\text { Cumulative } \\
\text { Percent }\end{array}$ \\
\hline \multirow{5}{*}{ Valid } & & 4 & 4.4 & 4.4 & 4.4 \\
\hline & $<200 \mathrm{ml}$ & 11 & 12.1 & 12.1 & 16.5 \\
\hline & $>400 \mathrm{ml}$ & 42 & 46.2 & 46.2 & 62.6 \\
\hline & $200-400 \mathrm{ml}$ & 34 & 37.4 & 37.4 & 100.0 \\
\hline & Total & 91 & 100.0 & 100.0 & \\
\hline
\end{tabular}

\begin{tabular}{|l|l|l|l|l|l|}
\hline \multicolumn{2}{|l|}{ why you drinks this caffeinated drinks } & Frequency & Percent & Valid Percent & $\begin{array}{l}\text { Cumulative } \\
\text { Percent }\end{array}$ \\
\hline \multirow{4}{*}{ Valid } & 2 & 2.2 & 2.2 & 2.2 \\
\cline { 2 - 6 } & Due to eat meal & 9 & 9.9 & 9.9 & 12.1 \\
\cline { 2 - 6 } & change my mode & 1 & 1.1 & 1.1 & 13.2 \\
\cline { 2 - 6 } & $\begin{array}{l}\text { Due to physical } \\
\text { headache }\end{array}$ & 4 & 4.4 & 4.4 & 17.6 \\
\cline { 2 - 6 } & Due to placement & 2 & 2.2 & 2.2 & 19.8 \\
\cline { 2 - 6 } & Due to Social life & 47 & 51.6 & 51.6 & 71.4 \\
\cline { 2 - 5 } $\begin{array}{l}\text { Due to studying } \\
\text { concentration }\end{array}$ & 25 & 27.5 & 27.5 & 98.9 \\
\cline { 2 - 6 } & i dont & 1 & 1.1 & 1.1 & 100.0 \\
\cline { 2 - 6 } & Total & 91 & 100.0 & 100.0 & \\
\hline
\end{tabular}

\begin{tabular}{|c|c|c|c|c|c|}
\hline \multicolumn{6}{|c|}{ do you feel any of this symptoms when stop this caffeinated drinks } \\
\hline & & Frequency & Percent & Valid Percent & $\begin{array}{l}\text { Cumulative } \\
\text { Percent }\end{array}$ \\
\hline \multirow[t]{21}{*}{ Valid } & & 13 & 14.3 & 14.3 & 14.3 \\
\hline & Fast heartbeat & 2 & 2.2 & 2.2 & 16.5 \\
\hline & headache & 1 & 1.1 & 1.1 & 17.6 \\
\hline & Headache & 1 & 1.1 & 1.1 & 18.7 \\
\hline & i dont drink it & 1 & 1.1 & 1.1 & 19.8 \\
\hline & Insomnia & 22 & 24.2 & 24.2 & 44.0 \\
\hline & Irritability & 6 & 6.6 & 6.6 & 50.5 \\
\hline & Nervousness & 8 & 8.8 & 8.8 & 59.3 \\
\hline & no & 1 & 1.1 & 1.1 & 60.4 \\
\hline & No & 1 & 1.1 & 1.1 & 61.5 \\
\hline & no symptoms & 1 & 1.1 & 1.1 & 62.6 \\
\hline & non & 1 & 1.1 & 1.1 & 63.7 \\
\hline & none & 1 & 1.1 & 1.1 & 64.8 \\
\hline & nothing & 2 & 2.2 & 2.2 & 67.0 \\
\hline & Nothing & 1 & 1.1 & 1.1 & 68.1 \\
\hline & Restlessness & 15 & 16.5 & 16.5 & 84.6 \\
\hline & Stomach upset & 10 & 11.0 & 11.0 & 95.6 \\
\hline & لا يو جد اعراض & 2 & 2.2 & 2.2 & 97.8 \\
\hline & ليس هناك اعراض & 1 & 1.1 & 1.1 & 98.9 \\
\hline & و لاشيء & 1 & 1.1 & 1.1 & 100.0 \\
\hline & Total & 91 & 100.0 & 100.0 & \\
\hline
\end{tabular}



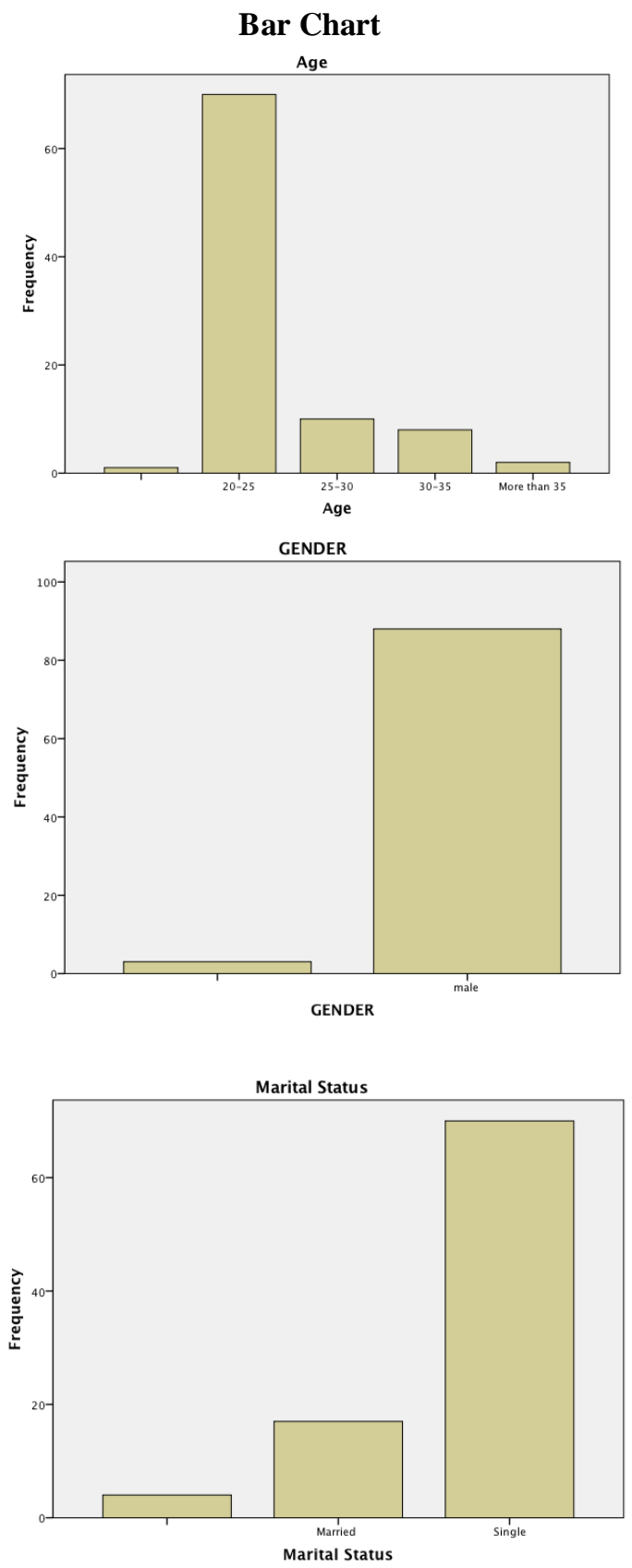

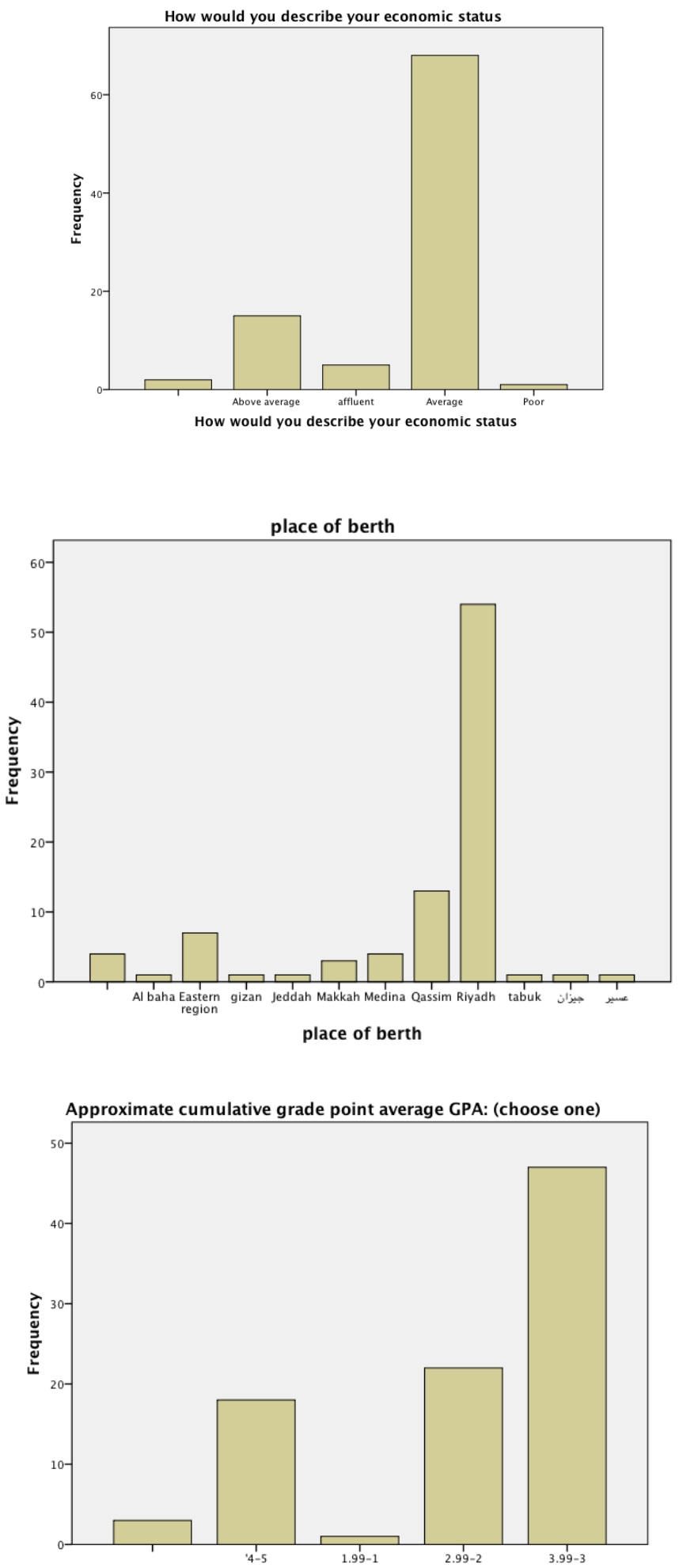

Approximate cumulative grade point average GPA: (choose one) 


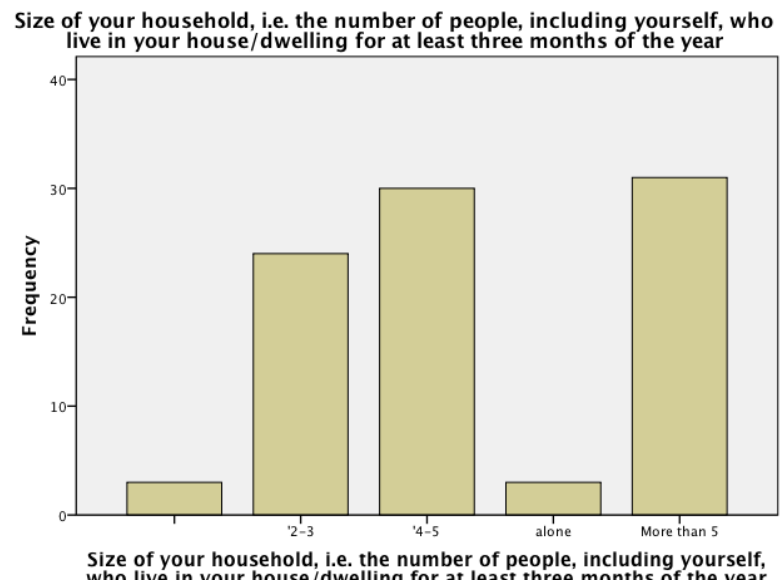
Sho of your household, i.e. the number of people, including yourself,

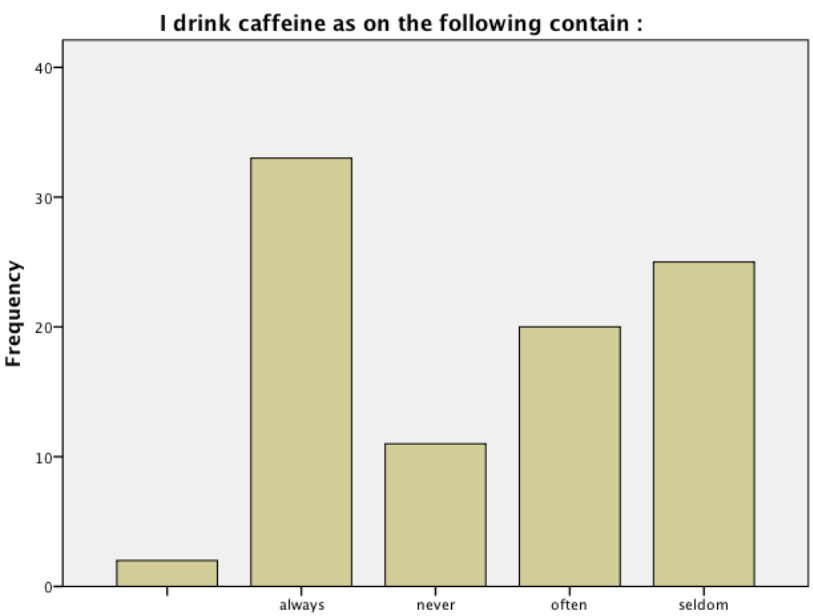

I drink caffeine as on the following contain :

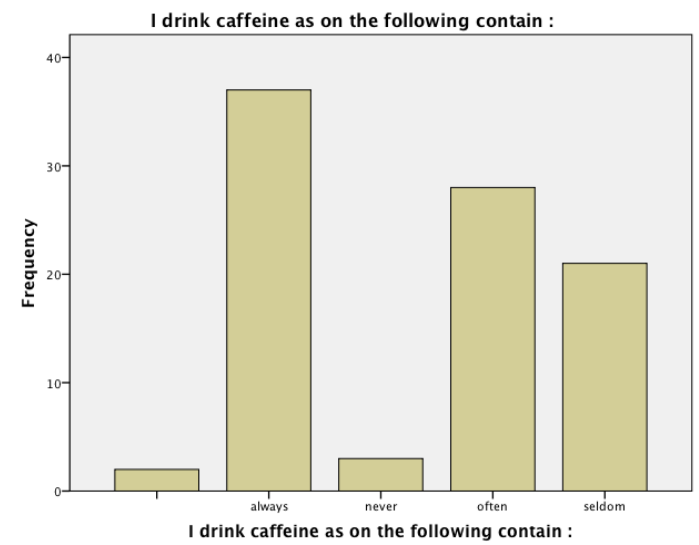




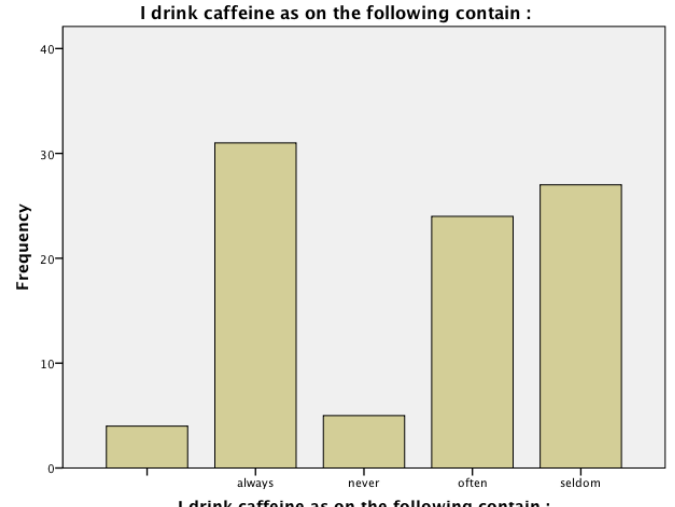

I drink caffeine as on the following contain :
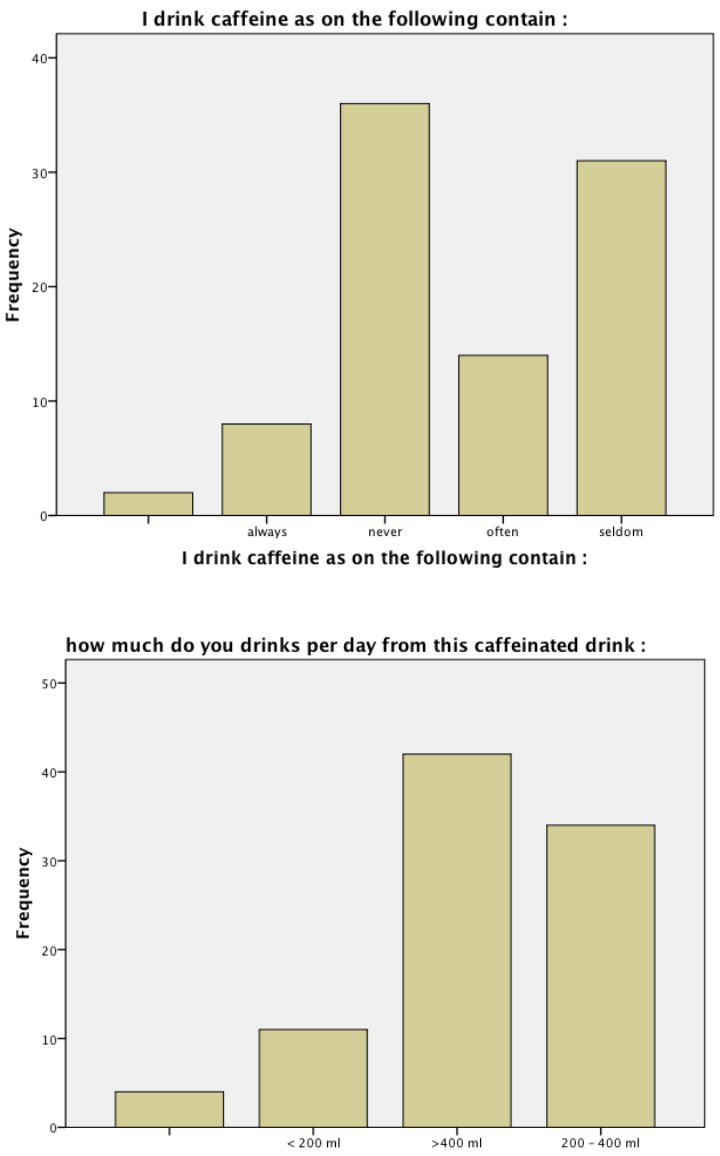

how much do you drinks per day from this caffeinated drink 

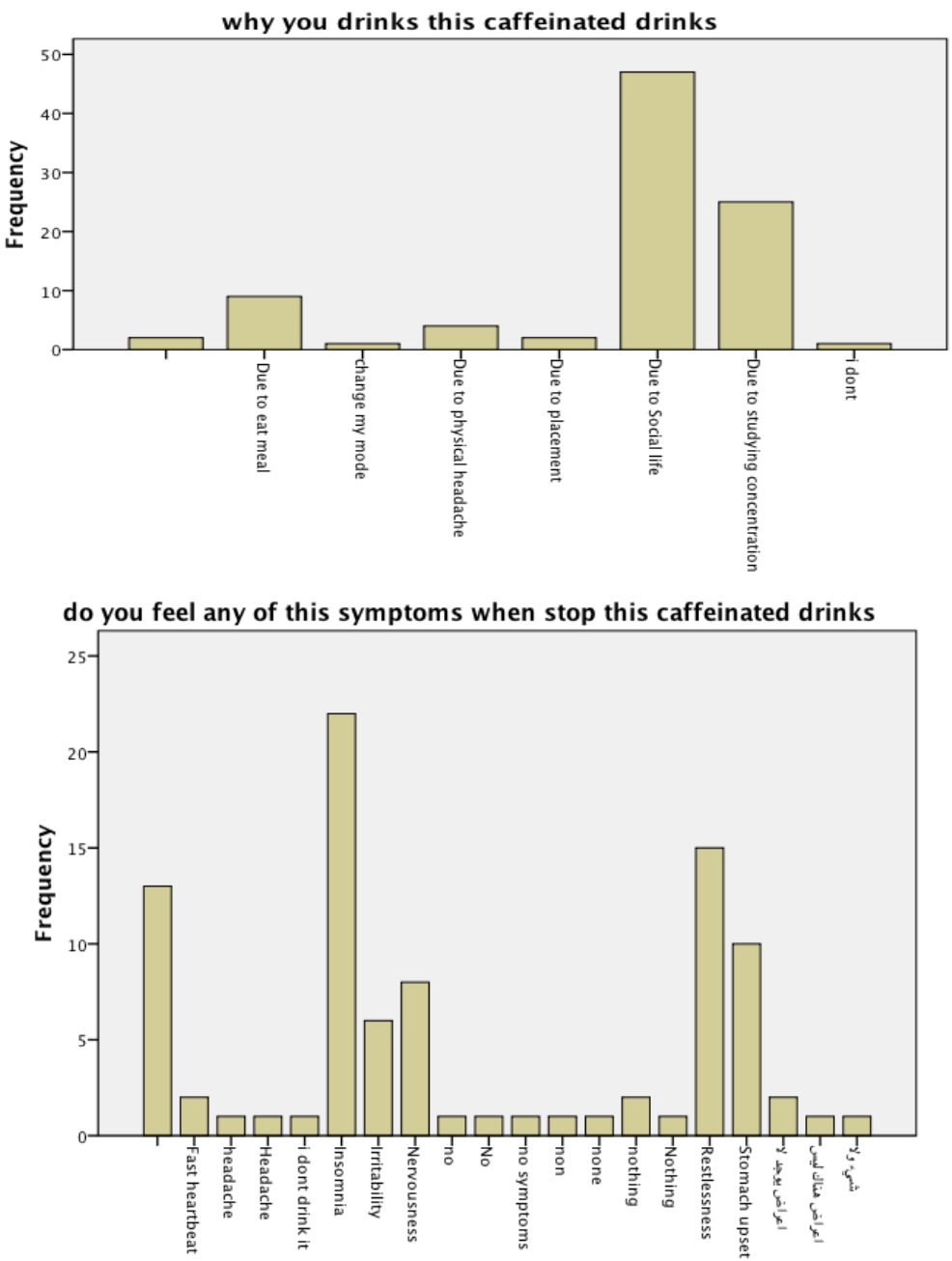

do you feel any of this symptoms when stop this caffeinated drinks 


\begin{tabular}{|c|c|c|c|c|c|c|}
\hline \multicolumn{7}{|c|}{ 1- I drink caffeinated coffee as on the following contain : } \\
\hline & & & & & & \\
\hline & & & & & & \\
\hline$<200 \mathrm{ml}$ & & & 1 & 2 & & 3 \\
\hline$>400 \mathrm{ml}$ & 1 & & 5 & 10 & & 16 \\
\hline $200-400 \mathrm{ml}$ & 5 & & 2 & 6 & & 13 \\
\hline \multirow[t]{2}{*}{ (blank) } & & & & 1 & & 1 \\
\hline & & & & & & \\
\hline$<200 \mathrm{ml}$ & & & & 1 & & 1 \\
\hline$>400 \mathrm{ml}$ & & & 2 & 4 & & 6 \\
\hline \multirow[t]{2}{*}{$200-400 \mathrm{ml}$} & & & 1 & 2 & & 3 \\
\hline & & & & & & \\
\hline$<200 \mathrm{ml}$ & 1 & & 1 & & & 2 \\
\hline$>400 \mathrm{ml}$ & 2 & & 2 & 5 & & 9 \\
\hline \multirow[t]{2}{*}{$200-400 \mathrm{ml}$} & 2 & 1 & 3 & 3 & & 9 \\
\hline & & & & & & \\
\hline$<200 \mathrm{ml}$ & 4 & & & 1 & & 5 \\
\hline$>400 \mathrm{ml}$ & & & 3 & 8 & & 11 \\
\hline $200-400 \mathrm{ml}$ & 2 & & 2 & 3 & 1 & 8 \\
\hline \multirow[t]{2}{*}{ (blank) } & 1 & & & & & 1 \\
\hline & & & & & & \\
\hline \multicolumn{7}{|l|}{ (blank) } \\
\hline Grand Total & 18 & 1 & 22 & 46 & 1 & 88 \\
\hline
\end{tabular}

\begin{tabular}{|c|c|c|c|c|c|c|}
\hline \multicolumn{7}{|c|}{ 2- I drink Tea drinks as on the following contain: } \\
\hline & & & & & & \\
\hline & & & & & & \\
\hline$<200 \mathrm{ml}$ & 1 & & & 2 & & 3 \\
\hline$>400 \mathrm{ml}$ & 1 & & 4 & 17 & & 22 \\
\hline $200-400 \mathrm{ml}$ & 3 & & 5 & 3 & & 11 \\
\hline (blank) & & & & 1 & & 1 \\
\hline & & & & & & \\
\hline$<200 \mathrm{ml}$ & & & & 1 & & 1 \\
\hline$>400 \mathrm{ml}$ & & & 1 & & & 1 \\
\hline $200-400 \mathrm{ml}$ & & & & 1 & & 1 \\
\hline & & & & & & \\
\hline$<200 \mathrm{ml}$ & & & 1 & & & 1 \\
\hline$>400 \mathrm{ml}$ & 1 & & 5 & 7 & & 13 \\
\hline $200-400 \mathrm{ml}$ & 4 & 1 & 2 & 5 & 1 & 13 \\
\hline$<200 \mathrm{ml}$ & 4 & & 1 & 1 & & 6 \\
\hline$>400 \mathrm{ml}$ & 1 & & 2 & 3 & & 6 \\
\hline $200-400 \mathrm{ml}$ & 2 & & 1 & 5 & & 8 \\
\hline (blank) & 1 & & & & & 1 \\
\hline (blank) & & & & & & \\
\hline Grand Total & 18 & 1 & 22 & 46 & 1 & 88 \\
\hline
\end{tabular}




\begin{tabular}{|c|c|c|c|c|c|c|}
\hline \multicolumn{7}{|c|}{ 3- I drink soft drinks as on the following contain : } \\
\hline$<200 \mathrm{ml}$ & & & 1 & & & 1 \\
\hline$>400 \mathrm{ml}$ & 2 & & 4 & 12 & & 18 \\
\hline $200-400 \mathrm{ml}$ & 2 & & 3 & 6 & & 11 \\
\hline (blank) & & & & 1 & & 1 \\
\hline & & & & & & \\
\hline$<200 \mathrm{ml}$ & 1 & & & 1 & & 2 \\
\hline $200-400 \mathrm{ml}$ & 1 & & & 1 & & 2 \\
\hline (blank) & 1 & & & & & 1 \\
\hline & & & & & & \\
\hline$<200 \mathrm{ml}$ & 1 & & & & & 1 \\
\hline$>400 \mathrm{ml}$ & 1 & & 4 & 10 & & 15 \\
\hline $200-400 \mathrm{ml}$ & 2 & & 2 & 3 & & 7 \\
\hline & & & & & & \\
\hline$<200 \mathrm{ml}$ & 3 & & 1 & 3 & & 7 \\
\hline$>400 \mathrm{ml}$ & & & 4 & 5 & & 9 \\
\hline $200-400 \mathrm{ml}$ & 3 & 1 & 3 & 3 & 1 & 11 \\
\hline $200-400 \mathrm{ml}$ & 1 & & & 1 & & 2 \\
\hline (blank) & & & & & & \\
\hline Grand Total & 18 & 1 & 22 & 46 & 1 & 88 \\
\hline
\end{tabular}

\begin{tabular}{|c|c|c|c|c|c|c|}
\hline \multicolumn{7}{|c|}{ 4- I drink energy drinks as on the following contain : } \\
\hline & & & & & & \\
\hline & & & & & & \\
\hline$>400 \mathrm{ml}$ & & & & 3 & & 3 \\
\hline \multirow[t]{2}{*}{$200-400 \mathrm{ml}$} & 3 & & 1 & 1 & & 5 \\
\hline & & & & & & \\
\hline$<200 \mathrm{ml}$ & 4 & & 2 & 2 & & 8 \\
\hline$>400 \mathrm{ml}$ & & & 4 & 7 & & 11 \\
\hline $200-400 \mathrm{ml}$ & 4 & 1 & 4 & 6 & 1 & 16 \\
\hline (blank) & 1 & & & & & 1 \\
\hline$>400 \mathrm{ml}$ & & & 6 & 6 & & 12 \\
\hline \multirow{2}{*}{$200-400 \mathrm{ml}$} & & & 1 & 1 & & 2 \\
\hline & & & & & & \\
\hline$<200 \mathrm{ml}$ & 1 & & & 2 & & 3 \\
\hline$>400 \mathrm{ml}$ & 3 & & 2 & 11 & & 16 \\
\hline $200-400 \mathrm{ml}$ & 2 & & 2 & 6 & & 10 \\
\hline \multirow[t]{2}{*}{ (blank) } & & & & 1 & & 1 \\
\hline \multirow{2}{*}{\multicolumn{7}{|c|}{ (blank) }} \\
\hline & & & & & & \\
\hline Grand Total & 18 & $\mathbf{1}$ & 22 & 46 & $\mathbf{1}$ & $\mathbf{8 8}$ \\
\hline
\end{tabular}

\section{Result:-}

There are 91 male medical students contributes in this study, $76.9 \%$ of the students their ages is between $20-25,11 \%$ their ages between $25-30,8.8 \%$ is $30-35$ and $2.2 \%$ is more than 35 years old. $18.7 \%$ of the students are married and 76.9 are not. The result of the economic status showing that $16.2 \%$ there economic status is above average, $5.5 \%$ affluent, 74.7 average and $1.1 \%$ is poor. $59 \%$ of the student their place of birth is Riyadh, $14 \%$ qassim, $4.4 \%$ madina , $7.7 \%$ in the eastern region, $3.3 \%$ in makkah, $1.1 \%$ is the percentage in Albaha, Gizan, Jeddah, Tabuk and Asser. 
The GPA percentage showing that $51.6 \%$ of the students their GPA is between (3-3.99), 24.2\% between (2-2.99), $19.8 \%(4-5)$, and $1.1 \%$ their GPA between( $1-1.99) .34 .1 \%$ they have more than 5 members at house, $33 \%$ between 4-5 members, $26.4 \%$ between 2-3 members, and 3.3\% they live alone.

$46.2 \%$ of the students drink more than $400 \mathrm{ml}$ of caffeinated drinks per day, 37.4\% 200-400 $\mathrm{ml}$ per day and 12.1\% less than $200 \mathrm{ml}$ per day.

$51.6 \%$ of the students drink caffeinated drinks due to social, 27.5 due to study, $9.9 \%$ due to meal, $4.4 \%$ due to physical headache and $1.1 \%$ they don't.

24.2 of the students they have insomnia, 16.5 have restlessness, $11 \%$ stomach upset, $8.8 \%$ nervousness , $6.6 \%$ irritability, $2.2 \%$ fast heart beat and the same percentage they don't have any symptoms, $1.1 \%$ headache.

Regarding the relationship between the type of the drinks and students grads, we found that the general GPA in the students who always drinks caffeinated drinks is 3.5 , and who never drinks is 3.5 , often 3.3 and finally seldom is 3.6 .

\section{Conclusion:-}

From the result we have in our hand and the comparison between the students GPA and the amount and the type of the caffeinated drinks we can see that there is do effected GPA because of the caffeine.

\section{Recommendation:-}

The effect of caffeine in medical student and how it affects the GPA need to be considered. The students may get health problems which can reflect on his effort of study and work after graduate.

\section{Refferences:-}

1. How caffeine affects test taking in college students by Diane Todd (May 06, 2010).

2. The relationship between caffeine consumption and study habits.

3. http://www.mancol.edu/stntlife/ccenter/articles/addicti/caff.html on October 17, 2000.

4. Sleep habits and their consequences: a survey umar a. khan, sara n. pasha, sarah k. khokhar, asim a. rizvi From department of physiology, shifa college of medicine, islamabad-pakistan(original article).

5. Tips for students - how to improve study habits during exams? : http://www.studyskillswebinar.com.

6. The relationship between caffeine consumption and the study habits and grades in college students. university of Kentucky.

7. Low-stress study tips for finals season by elizabeth scott, m.s., about.com guide.

8. Oct. 1, 2011 ( ) 1998-2013 Mayo Foundation for Medical Education and Research (MFMER). 